\title{
Respondents' report of a clinician- diagnosed depression in health surveys: comparison with DSM-IV mental disorders in the general adult population in Germany
}

Ulrike E. Maske ${ }^{1}$, Ulfert Hapke ${ }^{1 *}$, Steffi G. Riedel-Heller ${ }^{2}$, Markus A. Busch ${ }^{1}$ and Ronald C. Kessler ${ }^{3}$

\begin{abstract}
Background: Respondents' report of a previously diagnosed depression by a health professional is frequently used to estimate depression prevalence. This study contributes to a better understanding of survey results based on this measure by comparing it with a comprehensive standardized diagnostic interview.

Methods: Data came from the cross-sectional nationwide German Health Interview and Examination Survey for Adults (DEGS1) and its mental health module (DEGS1-MH, $n=4483$ ). In DEGS1, participants were asked whether they have been diagnosed with depression by a physician or psychotherapist (last 12-month). DSM-IV-based 12-month major depressive disorder (MDD) and other mental disorders were assessed with the German version of the Composite International Diagnostic Interview (CIDI). Time lag between both assessments was 6 weeks (median).

Results: $73.4 \%$ of participants reporting clinician-diagnosed depression met criteria for any mental disorder in the CIDI (any affective disorder: 51.8\%, any anxiety disorder: 54.7\%). The proportion of participants reporting a cliniciandiagnosed depression who met MDD criteria was highest among those aged 18-29 years (62.6\%) and decreased with age (65-79 years: 29.8\%). Among participants with MDD, the proportion with clinician-diagnosed depression was 33.0\%, highest among those aged 45-64 years (49.3\%) and lowest among those aged 18-29 years (22.7\%) and 30-44 years (20.3\%). MDD severity was positively associated with clinician-diagnosed depression.

Conclusions: Respondents' report of a clinician-diagnosed depression and major depression assessed with the CIDI substantially differ. Concordance of both measures varies with age and severity of depressive symptoms. Health surveys should assess a range of depression indicators in order to cover a wide spectrum.
\end{abstract}

Keywords: Depression, Diagnosis, Composite International Diagnostic Interview, General population, Health surveys

\section{Background}

In many large-scale health surveys, the prevalence of depression is assessed by asking participants whether they have been diagnosed with depression by a health professional in the past 12 months [1-4]. This simple measure has various preconditions such as previous health care utilization of the respondent, reporting depressive symptoms to a health professional, understanding the question asked in the survey, and admitting the diagnosis in

\footnotetext{
* Correspondence: FG26-psychische-Gesundheit@rki.de

${ }^{1}$ Department of Epidemiology and Health Monitoring, Division 26 Mental Health, Robert Koch Institute, Berlin, Germany

Full list of author information is available at the end of the article
}

the survey interview. In addition, the accuracy of this measure in determining the existence of depression depends on whether health professionals correctly diagnose their patient with depression. The latter can be at least partly questioned considering the notable extent of over- and under-diagnosis of depression in primary care found in clinical and community studies [5-8].

Despite these preconditions, a recent general population survey found that 12-month prevalence estimates based on the respondent's report of a clinician-diagnosis of depression and an interviewer assessment of major depression with a comprehensive standardized diagnostic interview based on the Diagnostic and Statistical 
Manual of Mental Disorders-IV (DSM-IV) were on a similar level [1]. Yet, the overlap of the two measures was only moderate, and, in agreement with previous research $[2,4,9,10]$, prevalence of major depression was highest in younger age adults and of clinician-diagnosed depression in middle-aged and older adults [1]. These findings suggest that there are substantial differences between DSM-IV-based major depression and a cliniciandiagnosed depression and, as a consequence, that the presence of major depression may not be inferred with accuracy from the respondent's report of a previous clinician diagnosis. However, there is a lack of specific knowledge about differences between both measures at an individual level.

Considering the fact that depression is still quite often assessed with a single question about clinician diagnoses in health surveys, this study aims to contribute to a better understanding of survey results based on the respondent's report of a clinician-diagnosed depression. Therefore, we investigate the association between such reports and independent diagnoses of depression in a large general population survey. Specifically, we examine the proportion of mental disorders based on a comprehensive standardized diagnostic research interview among survey participants who report a cliniciandiagnosed depression as a function of sex, age, and depression severity. Moreover, in order to examine the false negative rate based on the question about a previous clinician diagnosis, we examine the proportion of survey participants with major depression determined by the diagnostic interview who do versus do not report that a clinician diagnosed them with depression. Finally, socio-demographic, health-related and mental health characteristics are reported separately for survey participants with different combinations of depression diagnoses based on the cross-classification of diagnoses according to a diagnostic interview administered in the survey and the respondents' reports of being diagnosed by a clinician.

\section{Methods}

\section{Study design and sample}

Data come from the cross-sectional nationwide "German Health Interview and Examination Survey for Adults" (DEGS1) and its mental health module (DEGS1-MH; $n$ $=4483$; age $18-79$ years). The design, objectives and methods have been described in detail elsewhere [1114]. Briefly, a random sample of persons aged 18-79 years stratified for sex, age and geographical location was selected using two-stage clustered random sampling. On the first stage, 180 sample points were drawn from all German municipal communities, on the second stage, participants were randomly drawn from population registries of these sample points. For DEGS1-MH, all
DEGS1 participants with complete assessment (interview and examinations) aged 18-79 years were eligible who had consented to being re-contacted for the mental health module, who had sufficient language skills and who were available during the assessment period [11, 14]. In DEGS1, data were collected by self-administered written questionnaire and a standardized physicianadministered computer-assisted personal interview (CAPI) and physical and laboratory measurements. In DEGS1-MH, 12-month and lifetime diagnoses of mental disorders based on the diagnostic criteria of the DSMIV-TR were assessed in a CAPI using a modified version of the Composite International Diagnostic Interview (DIA-X/M-CIDI) [15, 16], a German version of the internationally established CIDI $[15,17,18]$. The median time lag between DEGS1 and DEGS1-MH was 6 weeks (inter quartile range 5-25 weeks). DEGS1 was approved by the federal and state commissioners for data protection and by the ethics committee of Charité-Universitätsmedizin Berlin (No.EA2/047/08). DEGS1-MH was additionally approved by the ethics committee of the Technische Universität Dresden (No.EK174062009). All participants provided written informed consent.

\section{Measures \\ Clinician-diagnosed depression based on the respondents' report}

Lifetime clinician-diagnosed depression was assessed in the physician-administered CAPI in DEGS1, where participants were asked: "Have you ever been diagnosed with depression by a physician or a psychotherapist?" If affirmed, 12-month clinician-diagnosed depression was determined with the question: "Was the depression present during the last 12 months?"

\section{Major depressive disorder (MDD) and other mental disorders based on a diagnostic interview}

MDD in the past 12 months was determined by the DIA-X/M-CIDI. In the DIA-X/M-CIDI, the presence of depression symptoms is assessed in 30 single items, which are grouped into the nine depression symptoms of DSM-IV based on a standardized algorithm. MDD was determined by applying all DSM-IV diagnostic criteria. Compared to a clinical lifetime diagnosis, a sensitivity of 0.95 (single depressive episode, lifetime) and 0.929 (recurrent major depressive episode, lifetime), and a specificity of 1.0 for both were reported [19]. In the same study, Kappa values of 0.82 for single major depression and 0.9 for recurrent major depression were found for the agreement of 1-month CIDI-based diagnosis and clinician-diagnosis. Substantial test-retest reliability has been shown for the M-CIDI [15].

Further 12-month mental disorders were assessed by the DIA-X/M-CIDI: other mood disorders (dysthymia, 
bipolar disorder I and II), anxiety disorders (panic disorder, agoraphobia, generalized anxiety disorder, social phobia, specific phobias, obsessive-compulsive disorder, post-traumatic stress disorder (PTSD)), somatoform disorders (pain disorder, undifferentiated somatoform disorder measured with the Somatic Symptom Index, SSI4,6; [20]), substance use disorders (alcohol and medication/drug abuse and dependence) without nicotine dependence, possible psychotic disorders (screening without further differential diagnosis), eating disorders (anorexia nervosa, bulimia nervosa, binge eating disorder) and mental disorder due to general medical conditions or substance induced disorders.

\section{Depression severity}

In addition to the presence of any comorbid mental disorder as described above excluding MDD, the following depression severity indicators were defined. For participants with MDD, the number of depression symptoms affirmed in the CIDI depression section was categorized into the following groups taking the lower and upper quartile as cut-offs: 5 symptoms (mild depression), 6-7 symptoms (moderate depression) and 8-9 symptoms (severe depression). Moreover, affirmation of thoughts about death or suicidal plan or attempt was established as a second CIDI-based severity indicator. Psychiatric comorbidity was determined for all participants based on all mental disorders assessed excluding MDD. Based on the self-administered German version of the Patient Health Questionnaire-9 (PHQ-9) [21, 22], which was assessed in all participants in DEGS1 and in DEGS1$\mathrm{MH}$, two additional severity indicators were defined: first, having depressive symptoms in both PHQ-9 assessments (i.e. sum score of 10 or more points in DEGS1 and DEGS1-MH) $[21,23]$ and second, having reported thoughts of being better off dead or of hurting oneself for at least several days in both PHQ-9 assessments.

\section{Socio-demographic and health related characteristics}

In DEGS1, sex, age, marital status, employment status and a range of further socio-demographic and healthrelated characteristics were assessed. Number of outpatient physician visits (excluding dentist visits) in the past 12 months was determined in a self-administered questionnaire based on a comprehensive list of medical disciplines. Including medical psychotherapist and psychological psychotherapist visits, the number of outpatient visits (minimum 0, maximum 155) was categorized into quartiles $(0-2,3-5,6-9, \geq 10)$ for analyses. Education was grouped into low, middle and high based on the Comparative Analysis of Social Mobility in Industrial Nations (CASMIN) [24]. Socio-economic status (SES) was classified as low, middle and high using an index based on information on education, occupational status and net household income [25]. Self-perceived social support was assessed using the Oslo-3 Social Support Scale and categorized as poor (3-8 points), moderate (9-11 points) and strong support (12-14 points) [26]. Community size was determined based on official administrative municipal codes for the place of residence. Health-related quality of life was examined using the physical and mental component scores of the Short Form 36 (SF-36) [27, 28].

\section{Statistical analyses}

Proportions are reported with 95\% confidence intervals $(95 \% \mathrm{CI})$. Pearson's $X^{2}$ test was used to determine whether two categorical variables were independent on a significance level of 0.05 . Associations were determined using bivariate and multivariate logistic regression models. To test for trend over the age groups, age group was included in the logistic regression model as a continuous variable. To analyse whether time lag between DEGS1 and DEGS1-MH has an influence on the results, the following analyses were carried out: Sensitivity analyses were conducted by excluding the upper quartile from the analyses (time lag to $>25$ weeks). Additionally, cross-tables showing the overlap of CIDI-based MDD and clinician-diagnosed depression stratified for the time lag (0-25 weeks vs. more than 25 weeks) can be found in the Additional file 1. Further, a logistic regression model with CIDI-based MDD as dependent variable and clinician-diagnosed depression as independent variable was calculated, including an interaction term of clinician-diagnosed depression and time lag (in quartiles). The interaction term was not significant $(p=$ 0.152).

Analyses were conducted with a weighting factor which 1) accounts for the complex sampling design (selection probability of the sample point and selection probability of the respondent within the sample point) and 2) corrects sample deviations from the population structure as of 31 Dec 2010 with regard to age group, sex, region, nationality, community type, education and participation in DEGS1-MH [11, 14, 29]. Confidence intervals were determined using STATA's survey procedures (STATA 14), which takes into account the weighting and the correlation of participants within a sample point. A non-responder analysis and a comparison with data from official statistics indicate that the DEGS1 sample is highly representative of the population aged 18-79 years in Germany [29].

\section{Results}

Of $n=4483$ participants of DEGS1-MH, $n=101$ (2.3\%) had missing values in at least one of both measures, resulting in a study sample of $n=4382$. In the sample, $50.9 \%(95 \% \mathrm{CI} \quad 48.9-52.9)$ were female, $19.2 \%(95 \% \mathrm{CI}$ 
17.9-20.5) were $18-29$ years old, $24.9 \%$ (95\%CI $23.3-$ 26.6) were $30-44$ years old, $36.0 \%$ (95\%CI $34.2-37.8$ ) were $45-64$ years old and $20.0 \%$ (95\% CI $18.7-21.3$ ) were $65-79$ years old. Overall, there were $78.8 \%$ (95\%CI 76.880.7) married and living with the partner or in a steady relationship. The distribution of CASMIN educational level was $35.0 \%(95 \% \mathrm{CI} 32.5-37.5)$ for low, $50.8 \%$ (95\%CI 48.7-52.9) for middle and $14.2 \%$ (95\%CI $12.6-$ 16.0) for high.

\section{Mental disorders among participants with a clinician- diagnosed depression}

Among those reporting a clinician-diagnosed depression, $73.4 \%$ had any mental disorder, of which anxiety disorders and affective disorders were the most frequent ones (Table 1). The proportion of all mental disorders was higher in participants with a clinician-diagnosed depression than in participants without a clinician-diagnosed depression in the past 12 months.

\section{MDD among participants with a clinician-diagnosed depression}

Among participants with a clinician-diagnosed depression, no substantial differences in the proportion of MDD and of any other mental disorder were found between men and women (Fig. 1). The percentage of participants with a clinician-diagnosed depression who also met the criteria of MDD was $62.6 \%$ in the age group of $18-29$ years (and 3.9\% remained without any mental disorder) and decreased to $29.8 \%$ in the age groups of $65-$ 79 years (and $39.8 \%$ remained without any mental disorder). The odds of meeting the criteria of MDD when reporting a clinician-diagnosed depression decreased per age group (OR: $0.6,95 \%$ CI $0.4-0.9 ; \mathrm{p}^{\text {trend }}=0.021$ ). The vast majority of those without clinician-diagnosed depression did not fulfil the criteria of MDD (men: 97.3\%,
95\%CI 96.3-98.0; women: 92.7\%, 95\%CI 90.9-94.2; $18-$ 29 years: $92.2 \%$, 95\%CI: $88.8-94.6$; $30-44$ years: $93.1 \%$, 95\%CI 90.1-95.2; 45-64 years: 96.7\%, 95\%CI 95.6-97.5; 65-79 years: 97.3, 95\%CI 95.5-98.4).

Among participants with a clinician-diagnosed depression, meeting the criteria of MDD was more likely with any other mental disorder present and with current depressive symptoms and the suicide item affirmed in both PHQ-9 assessments (Table 2).

\section{Clinician-diagnosed depression among participants with MDD}

Among participants with MDD, the proportion of a clinician-diagnosed depression did not differ between men and women, but it was lower in participants of younger than of older age (Table 3). Among those without MDD, the vast majority did not report a cliniciandiagnosed depression.

Among participants with MDD, clinician-diagnosed depression was less often reported by those aged 18-29 years (OR: $0.3,95 \% \mathrm{CI} 0.1-0.7 ; p=0.004$ ) and 30-44 years (OR: $0.3,95 \% \mathrm{CI} 0.1-0.8 ; p=0.014$ ) compared to the age group of 45-64 years. When adjusting for sex and the number of outpatient physician visits, these differences between the age groups, i.e. the different proportion of clinician-diagnosed depression among those with MDD, did not remain significant. In those aged 65-79 years with MDD, clinician-diagnosed depression was reported as often as by those aged $45-64$ years $(0.8$, 95\%CI 0.3-1.9; $p=0.641)$.

Among those with MDD, participants with any comorbid mental disorder and with severe MDD were more likely of reporting clinician-diagnosed depression (Table 4).

The only depression symptom associated with higher odds of reporting a clinician-diagnosed depression was

Table 1 Proportion of mental disorders among participants with and without a clinician-diagnosed depression

\begin{tabular}{|c|c|c|c|}
\hline & $\begin{array}{l}\text { 12-month clinician-diagnosed depression } \\
(n=249)\end{array}$ & $\begin{array}{l}\text { No 12-month clinician-diagnosed depression } \\
(n=4133)\end{array}$ & $p$ \\
\hline 12-month mental disorders & $\%(w)(95 \% C l)$ & $\%(w)(95 \% C l)$ & \\
\hline Any anxiety disorder ${ }^{\mathrm{a}}$ & $54.7(46.0-63.1)$ & $16.8(15.2-18.6)$ & $<0.0001$ \\
\hline Any affective disorder ${ }^{\mathrm{b}}$ & $51.8(42.9-60.5)$ & $7.4(6.4-8.5)$ & $<0.0001$ \\
\hline Possible psychotic disorder (screening) & $17.3(11.2-25.9)$ & $2.0(1.5-2.6)$ & $<0.0001$ \\
\hline Any substance use disorder ${ }^{c}$ & $15.2(10.7-21.2)$ & $5.4(4.4-6.5)$ & $<0.0001$ \\
\hline Any somatoform disorder ${ }^{d}$ & $14.8(10.4-20.7)$ & $3.0(2.4-3.8)$ & $<0.0001$ \\
\hline Any GMC/substance induced disorder & $7.7(3.4-16.4)$ & $0.8(0.5-1.2)$ & $<0.0001$ \\
\hline Any eating disorder ${ }^{\mathrm{e}}$ & $3.5(1.0-11.5)$ & $0.8(0.6-1.3)$ & 0.0227 \\
\hline Any of the above & $73.4(64.2-80.9)$ & $26.0(24.1-28.0)$ & $<0.0001$ \\
\hline
\end{tabular}

a including panic disorder, agoraphobia, generalized anxiety disorder, social phobia, specific phobias, obsessive-compulsive disorder, PTSD

b including major depression, dysthymia, bipolar disorder I or II

c including alcohol and medication abuse and dependence

d pain disorder and undifferentiated somatoform disorder as measured by the Somatic Symptom Index, SSI4,6 (Escobar et al., 1989)

e including anorexia nervosa, bulimia nervosa, binge eating disorder 


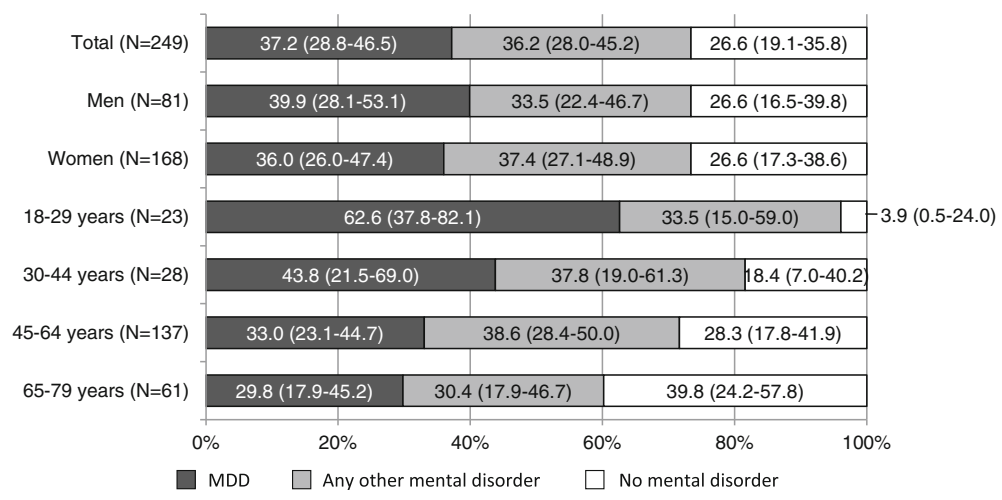

Fig. 1 Cumulative proportions of 12-month major depressive disorder (MDD) and any other mental disorder ${ }^{1}$ among participants with cliniciandiagnosed depression in the past 12 months. Including dysthymia, bipolar disorder I and II, panic disorder, agoraphobia, generalized anxiety disorder, social phobia, specific phobias, obsessive-compulsive disorder, PTSD, pain disorder, undifferentiated somatoform disorder, alcohol and medication abuse and dependence, possible psychotic disorders (screening without further differential diagnosis), anorexia nervosa, bulimia nervosa, binge eating disorder, mental disorder due to general medical conditions or substance induced disorders.

"thoughts of death or suicide, or having suicide plan or attempt" (results for other symptoms not shown).

\section{Socio-demographic, health-related and mental health characteristics of participants with a clinician-diagnosed depression and MDD, with a clinician-diagnosed depression only and MDD only}

Further analyses found significant overall differences of each socio-demographic characteristic considered between participants with clinician-diagnosed depression and MDD, with clinician-diagnosed depression only, with MDD only, and the non-cases according to both instruments (see Additional file 2). Participants with clinician-diagnosed depression who also met the criteria of MDD had the least favourable socio-demographic distributions, e.g. highest percentage of unmarried and without steady relationship, low SES, poor social support, never employed. Participants with cliniciandiagnosed depression with and without MDD had higher numbers of outpatient physician visits and of chronic somatic conditions and a lower physical health related quality of life than participants with MDD only. Mental health related quality of life was lowest among participants with clinician-diagnosed depression and MDD.

The proportion of mental disorders was the highest in participants with clinician-diagnosed depression who also met the criteria of MDD than in participants with a clinician-diagnosed depression only or MDD only, though not significantly throughout the disorders examined (see Additional file 3). Compared to participants with a clinician-diagnosed depression only and MDD only, those identified by both measures had a higher proportion of PHQ-9 current depressive symptoms and of PHQ-9 suicidal item affirmed in DEGS1 and DEGS1-MH.

Table 2 Proportion of major depressive disorder (MDD) among participants with clinician-diagnosed depression across depression severity indicators

\begin{tabular}{|c|c|c|c|c|c|c|}
\hline & \multicolumn{6}{|c|}{ Participants with a clinician-diagnosed depression in the past 12 months $(n=249)$} \\
\hline & 12-month MDD & No 12-month MDD & & & & \\
\hline & $\begin{array}{l}\text { Row } \\
\%(\mathrm{w})(95 \% \mathrm{Cl})\end{array}$ & $\begin{array}{l}\text { Row } \\
\%(w)(95 \% C l)\end{array}$ & $\begin{array}{l}\text { Bivariate OR } \\
(95 \% \mathrm{Cl})\end{array}$ & $p$ & $\begin{array}{l}\text { Adjusted OR } \\
(95 \% \text { Cl) }\end{array}$ & $p$ \\
\hline Any comorbid mental disorder ${ }^{\mathrm{b}}(N=171)$ & $48.0(37.0-59.2)$ & $52.0(40.8-63.0)$ & $6.4(2.4-17.2)$ & $<0.001$ & $5.8(2.2-15.3)$ & $<0.001$ \\
\hline No comorbid mental disorder $(N=78)$ & $12.6(5.9-25.0)$ & $87.4(75.0-94.1)$ & Ref. & & Ref. & \\
\hline Current depressive symptoms ${ }^{\mathrm{C}}(\mathrm{N}=64)$ & $63.4(46.9-77.3)$ & $36.6(22.7-53.1)$ & $4.6(2.3-9.5)$ & $<0.001$ & $4.6(2.2-9.5)$ & $<0.001$ \\
\hline No current depressive symptoms $(N=185)$ & $27.2(19.5-36.6)$ & $72.8(63.4-80.5)$ & Ref. & & Ref. & \\
\hline Affirmed suicide item ${ }^{d}(N=52)$ & $63.9(45.5-79.0)$ & $36.1(21.0-54.5)$ & $4.2(1.7-10.0)$ & 0.002 & $4.1(1.7-10.1)$ & 0.002 \\
\hline Suicide item not affirmed $(N=197)$ & $29.8(21.4-40.0)$ & $70.2(60.0-78.6)$ & Ref. & & Ref. & \\
\hline
\end{tabular}

adjusted for sex and age

b dysthymia, bipolar disorder I and II, panic disorder, agoraphobia, generalized anxiety disorder, social phobia, specific phobias, obsessive-compulsive disorder, PTSD, pain disorder, undifferentiated somatoform disorder, alcohol and medication abuse and dependence, possible psychotic disorders (screening without further differential diagnosis), anorexia nervosa, bulimia nervosa, binge eating disorder, mental disorder due to general medical conditions or substance induced disorders c PHQ-9 current depressive symptoms (sum score $\geq 10$ ) in DEGS1 and DEGS1-MH

${ }^{d}$ PHQ-9 suicide item affirmed (at least several days) in DEGS1 and DEGS1-MH 
Table 3 Proportion of participants with and without cliniciandiagnosed depression among participants with and without major depressive disorder (MDD)

\begin{tabular}{lll}
\hline & $\begin{array}{l}\text { 12-month clinician-diagnosed } \\
\text { depression in participants } \\
\text { with 12-month MDD }\end{array}$ & $\begin{array}{l}\text { No 12-month clinician- } \\
\text { diagnosed depression } \\
\text { in participants without } \\
\text { 12-month MDD } \\
\%(w)(95 \% C l)\end{array}$ \\
\hline Total & $33.0(25.9-40.9)$ & $95.8(94.8-96.6)$ \\
Sex & $37.1(26.0-49.7)$ & $97.6(96.6-98.3)$ \\
Men & $31.3(22.9-41.1)$ & $94.0(92.1-95.5)$ \\
Women & & \\
Age group (years) & $98.5(96.8-99.3)$ \\
18-29 years & $22.7(12.4-37.9)$ & $97.6(96.1-98.6)$ \\
30-44 years & $20.3(8.8-40.3)$ & $93.6(91.4-95.4)$ \\
45-64 years & $49.3(38.3-60.4)$ & $95.0(92.7-96.6)$ \\
$65-79$ years & $44.2(26.5-63.5)$ &
\end{tabular}

\section{Discussion}

Based on a nationally representative sample of the general adult population, this study shows that a large proportion of survey participants who report a previously clinician-diagnosed depression meets criteria of at least one DSM-IV mental disorder. At the same time, there were major differences between participants reporting a clinician-diagnosed depression and participants who meet criteria for major depression in a comprehensive standardized diagnostic interview.

The findings of this study should be considered in the context of several limitations. First, the time lag between DEGS1 and DEGS1-MH might have led to an underestimation of the overlap of both measures examined and of the prevalence of mental disorders among participants with a clinician-diagnosed depression due to remission or to the time difference between both assessments. Analyses limiting the time lag to $\leq 25$ weeks led to a slight increase of overlap, but the results did not substantially change. The time-lag stratified analyses of the overlap of both measures indicates that the time lag might have led to an age- or sex-specific under- or overestimation of the overlap. However, the confidence intervals are large due to the small numbers of cases in the upper quartile. Second, the measure of a cliniciandiagnosed depression is diffuse in two ways. The wording of the question leaves it unclear whether the depression diagnosis was made in the past 12 months or whether the respondent had received a diagnosis previously and interprets depressive symptoms in the past 12 months as a continuation. Further, the measure depends on the respondent's ability to recall a depression diagnosis, which however also applies for symptom reports in the CIDI. Third, the mental disorders assessed in DEGS1-MH does not include all possible disorders, hence the prevalence of any other mental disorder might be underestimated. Fourth, the small number of cases results in large confidence intervals and restricts analyses with respect to the number of strata.

Despite these limitations, this study contributes to a better understanding of survey results based on the measure of a clinician-diagnosed depression. There are four noteworthy findings. First, there was a remarkable age pattern: Among participants with a cliniciandiagnosed depression, the proportion of those meeting

Table 4 Proportion of clinician-diagnosed depression among participants with major depressive disorder (MDD) across depression severity indicators

\begin{tabular}{|c|c|c|c|c|c|c|}
\hline & \multicolumn{6}{|c|}{ Participants who met the criteria for 12 -month MDD $(n=284)$} \\
\hline & \multirow{2}{*}{$\begin{array}{l}\text { 12-month clinician- } \\
\text { diagnosed depression } \\
\text { Row } \\
\%(\mathrm{w})(95 \% \mathrm{Cl})\end{array}$} & \multicolumn{5}{|l|}{$\begin{array}{l}\text { No 12-month clinician- } \\
\text { diagnosed depression }\end{array}$} \\
\hline & & $\begin{array}{l}\text { Row } \\
\%(w)(95 \% C l)\end{array}$ & Bivariate OR $(95 \% \mathrm{Cl})$ & $p$ & Adjusted OR $(95 \% \mathrm{Cl})$ & $p$ \\
\hline Any comorbid mental disorder ${ }^{\mathrm{b}}$ & $37.9(29.4-47.3)$ & $62.1(52.7-70.6)$ & $3.3(1.3-8.5)$ & 0.012 & $6.6(1.7-25.6)$ & 0.006 \\
\hline No comorbid mental disorder ${ }^{\mathrm{b}}$ & $15.5(7.4-29.8)$ & $84.5(70.2-92.6)$ & Ref. & & Ref. & \\
\hline \multicolumn{7}{|l|}{ Number of depression symptoms } \\
\hline Mild (5/9 symptoms) & $19.0(10.5-31.9)$ & $81.0(68.1-89.5)$ & Ref. & & Ref. & \\
\hline Moderate (6\&7/9 symptoms) & $27.2(19.4-36.7$ & $72.8(63.3-80.6)$ & $1.6(0.7-3.7)$ & 0.275 & $2.3(0.9-5.4)$ & 0.066 \\
\hline Severe (8\&9/9 symptoms) & $49.5(34.4-64.7)$ & $50.5(35.3-65.6)$ & $4.2(1.7-10.4)$ & 0.002 & $7.0(2.6-19.0)$ & $<0.001$ \\
\hline $\begin{array}{l}\text { Thoughts of death or suicide, or } \\
\text { having suicide plan or attempt }\end{array}$ & $41.8(32.3-52.0)$ & $58.2(48.0-67.7)$ & $3.6(1.8-7.3)$ & $<0.001$ & $3.2(1.4-7.4)$ & 0.006 \\
\hline $\begin{array}{l}\text { No thoughts of death or suicide, } \\
\text { or having suicide plan or attempt }\end{array}$ & $16.5(10.2-25.6)$ & $83.5(74.4-89.8)$ & Ref. & & Ref. & \\
\hline
\end{tabular}

adjusted for sex, age groups, number of outpatient physician visits

${ }^{b}$ dysthymia, bipolar disorder I and II, panic disorder, agoraphobia, generalized anxiety disorder, social phobia, specific phobias, obsessive-compulsive disorder, PTSD, pain disorder, undifferentiated somatoform disorder, alcohol and medication abuse and dependence, possible psychotic disorders (screening without further differential diagnosis), anorexia nervosa, bulimia nervosa, binge eating disorder, mental disorder due to general medical conditions or substance induced disorders c based on the CIDI depression section 
the criteria of MDD was $62.6 \%$ in those aged 18-29 years and decreased per age group to $29.8 \%$ in those aged $65-79$ years. A similar yet less pronounced age pattern was found in a large community-based study in the US [5]. Interestingly, our results show that $96.1 \%$ of young adults who report a clinician-diagnosed depression had any mental disorder, while $39,8 \%$ of adults aged 65-79 years did not have any disorder even though reporting a health professional-diagnosed depression. The relatively high proportion of $62.6 \%$ MDD among those aged 18-29 years is higher than the $42.0 \%$ that was reported in a meta-analysis on the accuracy of unassisted depression diagnosis in primary care [30] and equal to the proportion of $66.5 \%$ which was reported for the optimal threshold for the CIDI screening scale [31]. Our study indicates that a clinician-diagnosed depression reported by this age group in a health survey might include to a large part participants who actually fulfilled the MDD criteria. A possible explanation for this finding is the higher prevalence of severe depression among depressed young adults [10] which might lead to more accurate diagnoses by health professionals. Also, health professionals might be more rigorous in diagnosing depression when it comes to younger patients because of its possible psychological impact on the life young people. Further, the high prevalence of depression in young adults $[9,10,14,32]$ might result in a higher number of "true positives" than in populations with lower depression prevalence. The high proportion of middle-aged and older participants with a cliniciandiagnosed depression who did not meet the criteria of MDD implies a substantial amount of over-diagnosis of depression for these groups, replicating results from primary care in Italy [6] and the US [5]. Increased physical complaints in older age could be misinterpreted as symptoms of depression by health professionals [33, 34]. Additionally, underreporting of depressive symptoms by older participants in the CIDI due to its complex questioning and its multiple time frames [35] and due to problems recalling the symptoms are possible methodological explanations. Research has suggested that older adults more frequently show clinically significant depressive symptoms without fulfilling all diagnostic criteria [36]. In this context, our finding could at least partly be explained by the fact that the depression diagnosis based on the CIDI might exclude older people due to the diagnostic algorithm who might have reported significant symptoms to the health professional, resulting in a depression diagnosis.

The second notable result is the striking age pattern of the proportion of clinician-diagnosed depression among participants with MDD (low in young age groups and highest in those aged 45-64 years). Obviously, these findings cannot be interpreted as the percentage of actual unrecognition of depression by health professionals. Instead, it reflects the specific preconditions of the measure of a clinician-diagnosed depression reported by the respondent. Thus, our findings supposedly mirror to a large part the lack of any health professional contact in people with mood disorders [37] and the fact that young adults are less likely to use professional health services due to mental health problems [38-40] than older adults, but prefer seeking help rather from family or friends [41]. Additionally, low numbers of physician contacts are reported for younger adults [42], reducing the odds for a physician to detect depression $[8,43]$. This is supported by the fact that the age pattern disappeared after controlling for the number of outpatient physician visits. In contrast to the US, where financial barriers have been reported the most common reasons for not seeking professional help for mental health problems [44], personal help-seeking barriers such as selfand perceived stigmatization [45-47] are likely more relevant in Germany, as diagnosis and treatment of mental disorders are covered by mandatory health insurance. As to depression recognition itself, our results reflect the considerable amount of unrecognized depression in primary care in Germany and internationally [8, 30, 48], which was suggested of being the most pronounced in younger people [7]. In addition, health professionals might not always fix and communicate a depression diagnosis to the patient [49]. Not least, selfstigmatization might lead to a reporting bias of a depression diagnosis in the survey interview.

Third, it is remarkable that no differences were found between men and women. On one hand, concerning the similar level of the proportion of MDD among men and women with a clinician-diagnosed depression, our results agree with findings from the US [5]. On the other hand, the fact that we did not find any sex difference regarding the proportion of clinician-diagnosed depression among those with MDD is contra-intuitive as research has suggested sex-specific barriers to help seeking [50], utilization of somatic and mental health services [42, 51], symptom reporting [52] and depression recognition rates [8].

Fourth, we found that participants with severe depression according to different severity indicators were more likely to be classified as cases by both measures. This is plausible considering that research found an association of seriousness of mental disorder with service use [38] and higher proportions of depression recognition in primary care when depression was severe and when suicidal ideation or tendency was reported by the patient [8]. This finding indicates that a considerable part of participants with severe depression is included in the measure of a clinician-diagnosed depression. In addition to the explanations given above, this finding 
can also be interpreted in the context of discussions about the DSM-IV potentially assigning a depression diagnosis partly to people without relevant disability [53]. Following this discourse, participants with depression without relevant disabilities might have qualified for a depression diagnosis in the CIDI, but were unlikely to seek for professional help due to their lack of disability.

\section{Conclusions}

This study contributes to a better understanding of survey results based on the respondent's report of a clinician-diagnosed depression. It shows that a large proportion of survey participants who report a cliniciandiagnosed depression meets criteria of at least one DSM-IV mental disorder (most frequently any affective and any anxiety disorder) according to the CIDI. However, substantial differences exist between participants who report a clinician-diagnosed depression and participants who meet criteria for depression in a comprehensive standardized diagnostic interview. Furthermore, concordance of both measures varies with age and severity of depressive symptoms. Thus, respondent reports of clinician-diagnosed depression cannot be considered a short alternative for CIDI-based major depression in studies of the general population. This needs to be considered when interpreting survey results based on reports about clinician-diagnosed depression.

Instead, this study underlines the importance to assess a range of depression indicators in health surveys in order to cover a wide spectrum. For many research questions, information about depression diagnosis in the health care system is important. But if information on depressive symptoms or on the presence of diagnostic criteria is needed, further indicators such as standardized diagnostic interviews or one of the established short screening scales, which have been shown to have good concordance with diagnoses based on independent research diagnostic interviews [21,31], should be additionally assessed.

Regarding clinical practice, our findings first imply that if patients report that they have not been diagnosed with depression, they most likely do neither have a history of major depression. Second, relating to younger patients, health professionals should be especially attentive and keep a possible major depression in mind when depressive symptoms are reported in order to provide adequate treatment. Third, relating to older patients, our results imply that health professionals should on one hand be more rigorous when diagnosing depression in order to reduce over-diagnosis and over-treatment as a consequence. On the other hand, however, health professionals should consider determining a differential depression diagnosis when depressive symptoms are reported by elderly in order to increase recognition and adequate depression treatment in this age group.

\section{Additional files}

Additional file 1: Proportions of 12-month clinician-diagnosed depression and CIDI-based 12-month major depression (MDD), stratified for time lag. (DOCX 24 kb)

Additional file 2: Socio-demographic and health-related characteristics of participants who report a 12-month clinician-diagnosed depression who did or did not meet the criteria for 12-month major depressive disorder (MDD). (DOCX 25 kb)

Additional file 3: Mental health characteristics of participants who report a 12-month clinician-diagnosed depression who did or did not meet the criteria for 12-month major depressive disorder (MDD). (DOCX $22 \mathrm{~kb}$ )

\section{Abbreviations}

CIDI: Composite International Diagnostic Interview; DEGS1: German Health Interview and Examination Survey for Adults; DEGS1-MH: Mental health module of the German Health Interview and Examination Survey for Adults; DSM-IV: Diagnostic and Statistical Manual of Mental Disorders, 4th edition; GNHIES98: German National Health Interview and Examination Survey 1998; MDD: Major depressive disorder; PHQ-9: Patient Health Questionnaire-9; SES: Socio-economic status

\section{Acknowledgements}

Data collection of DEGS1 and DEGS1-MH was funded primarily by the German Ministry of Health (BMG). Supplementary funding for DEGS1-MH was provided by the Technische Universität Dresden and by the Stiftung Seelische Gesundheit inaugurated by the German Association for Psychiatry and Psychotherapy (DGPPN). The German Academic Exchange Service (DAAD) granted a research stay of Ulrike Maske at Dr. Kessler's department. The funding sources had no involvement in the study design, in the collection, analysis and interpretation of data, in the writing of the report, and in the decision to submit the article for publication. We wish to thank Ingeburg Seiffert for helping to prepare and deliver data. Further, we thank all the study participants.

\section{Funding}

Data collection of DEGS1 and DEGS1-MH was funded primarily by the German Ministry of Health (BMG). Supplementary funding for DEGS1-MH was provided by the Technische Universität Dresden and by the Stiftung Seelische Gesundheit inaugurated by the German Association for Psychiatry and Psychotherapy (DGPPN). A three-month research stay of UEM at Dr. Kessler's department was funded by the German Academic Exchange Service (DAAD). The funding sources had no involvement in the study design, in the collection, analysis and interpretation of data, in the writing of the report, and in the decision to submit the article for publication.

\section{Availability of data and materials}

The authors confirm that some access restrictions apply to the data underlying the findings. The data set cannot be made publicly available because informed consent from study participants did not cover public deposition of data. However, the minimal data set underlying the findings is archived in the 'Health Monitoring' Research Data Centre at the Robert Koch Institute (RKI) and can be accessed by all interested researchers. On-site access to the data set is possible at the Secure Data Center of the RKI's 'Health Monitoring' Research Data Centre. Requests should be submitted to the 'Health Monitoring' Research Data Centre, Robert Koch Institute, Berlin, Germany (e-mail: fdz@rki.de)

\section{Authors' contributions}

UEM prepared and analyzed the data and designed and drafted the manuscript. RCK substantially contributed to the concept of the manuscript and data analyses and extensively helped writing the manuscript and interpreting the results. UH, SRH and MB helped drafting the manuscript and 
contributed to the interpretation. All authors read and approved the final manuscript.

\section{Competing interests}

In the past three years, Dr. Kessler has been a consultant for Hoffman-La Roche, Inc. and Johnson \& Johnson Wellness and Prevention. Dr. Kessler has served on advisory boards for Mensante Corporation, Johnson \& Johnson Services Inc. Lake Nona Life Project, and U.S. Preventive Medicine. Dr. Kessler is a co-owner of DataStat, Inc.

The remaining authors declare that they have no competing interests.

\section{Consent for publication}

Not applicable.

\section{Ethics approval and consent to participate}

DEGS1 was approved by the federal and state commissioners for data protection and by the ethics committee of Charité-Universitätsmedizin Berlin (No.EA2/047/08). DEGS1-MH was additionally approved by the ethics committee of the Technische Universität Dresden (No.EK174062009). All participants provided written informed consent prior to the assessment Participants were informed about the goals and contents of the study, about privacy and data protection, and that their participation in the study was voluntary.

\section{Author details}

'Department of Epidemiology and Health Monitoring, Division 26 Mental Health, Robert Koch Institute, Berlin, Germany. ${ }^{2}$ Institute of Social Medicine, Occupational Health and Public Health (ISAP), Faculty of Medicine, University of Leipzig, Leipzig, Germany. ${ }^{3}$ Department of Health Care Policy, Harvard University, Boston, MA, USA.

Received: 2 June 2016 Accepted: 10 January 2017

Published online: 23 January 2017

\section{References}

1. Maske UE, Buttery AK, Beesdo-Baum K, Riedel-Heller S, Hapke U, Busch MA Prevalence and correlates of DSM-IV-TR major depressive disorder, selfreported diagnosed depression and current depressive symptoms among adults in Germany. J Affect Disord. 2016;190:167-77.

2. Strine TW, Mokdad AH, Balluz LS, Gonzalez O, Crider R, Berry JT, Kroenke K. Depression and anxiety in the United States: findings from the 2006 Behavioral Risk Factor Surveillance System. Psychiatr Serv. 2008:59(12):1383-90.

3. Substance Abuse and Mental Health Services Administration: Summary of National Findings. In: Results from the 2010 National Survey on Drug Use and Health. Rockville: Substance Abuse and Mental Health Services Administration, editor; 2011.

4. Maske UE, Scheidt-Nave C, Busch MA, Jacobi F, Weikert B, Riedel-Heller SG, Hapke U. Komorbidität von Diabetes mellitus und Depression in Deutschland. Psychiatr Prax. 2015:42(4):202-7.

5. Mojtabai R. Clinician-identified depression in community settings: concordance with structured-interview diagnoses. Psychother Psychosom. 2013;82(3):161-9.

6. Berardi D, Menchetti M, Cevenini N, Scaini S, Versari M, De Ronchi D. Increased recognition of depression in primary care. Comparison between primary-care physician and ICD-10 diagnosis of depression. Psychother Psychosom. 2005:74(4):225-30.

7. Wittchen HU, Hofler M, Meister W. Prevalence and recognition of depressive syndromes in German primary care settings: poorly recognized and treated? Int Clin Psychopharmacol. 2001;16(3):121-35.

8. Wittchen HU, Pittrow D. Prevalence, recognition and management of depression in primary care in Germany: the Depression 2000 study. Hum Psychopharmacol. 2002;17 Suppl 1:1-11.

9. Kessler RC, Birnbaum H, Bromet E, Hwang I, Sampson N, Shahly V. Age differences in major depression: results from the National Comorbidity Survey Replication (NCS-R). Psychol Med. 2010:40(2):225-37.

10. Kessler RC, Birnbaum HG, Shahly V, Bromet E, Hwang I, McLaughlin KA Sampson N, Andrade LH, de Girolamo G, Demyttenaere K, et al. Age differences in the prevalence and co-morbidity of DSM-IV major depressive episodes: results from the WHO World Mental Health Survey Initiative. Depress Anxiety. 2010;27(4):351-64
11. Jacobi F, Mack S, Gerschler A, Scholl L, Hofler M, Siegert J, Burkner A, Preiss $\mathrm{S}$, Spitzer K, Busch $\mathrm{M}$, et al. The design and methods of the mental health module in the German Health Interview and Examination Survey for Adults (DEGS1-MH). Int J Methods Psychiatr Res. 2013;22(2):83-99.

12. Scheidt-Nave C, Kamtsiuris P, Goesswald A, Hoelling H, Lange M, Busch MA, Dahm S, Doelle R, Ellert U, Fuchs J, et al. German health interview and examination survey for adults (DEGS) - design, objectives and implementation of the first data collection wave. BMC Public Health. 2012; 12(1):730

13. Gößwald A, Lange $M$, Dölle $R$, Hölling $H$. The first wave of the German Health Interview and Examination Survey for Adults (DEGS1). Recruitment of participants, fieldwork, and quality assurance. Bundesgesundheitsblatt Gesundheitsforschung Gesundheitsschutz. 2013:56:611-9.

14. Jacobi F, Höfler M, Siegert J, Mack S, Gerschler A, Scholl L, Busch M, Hapke U, Maske U, Gaebel W, et al. Twelve-months prevalence of mental disorders in the German Health Interview and Examination Survey for Adults - Mental Health Module (DEGS1-MH): a methodological addendum and correction. Int J Methods Psychiatr Res. 2015;24(4):305-13.

15. Wittchen HU, Lachner $G$, Wunderlich U, Pfister $H$. Test-retest reliability of the computerized DSM-IV version of the Munich-Composite International Diagnostic Interview (M-CIDI). Soc Psychiatry Psychiatr Epidemiol. 1998; 33(11):568-78

16. Wittchen HU, Pfister H. DIA-X-Interviews: Manual für Screening-Verfahren und Interview; Interviewheft Längsschnittuntersuchung (DIA-X-Lifetime); Ergänzungsheft (DIA-X-Lifetime); Interviewheft Querschnittuntersuchung (DIA-X-12 Monate); Ergänzungsheft (DIA-X-12Monate); PC-Programm zur Durchführung des Interviews (Längs-und Querschnittuntersuchung); Auswertungsprogramm. Frankfurt: Swets und Zeitlinger; 1997.

17. Kessler RC, Ustun TB. The World Mental Health (WMH) Survey Initiative Version of the World Health Organization (WHO) Composite International Diagnostic Interview (CIDI). Int J Methods Psychiatr Res. 2004;13(2):93-121.

18. Wittchen $\mathrm{H}-\mathrm{U}$. Reliability and validity studies of the WHO-Composite International Diagnostic Interview (CIDI): a critical review. J Psychiatr Res. 1994:28(1):57-84.

19. Reed V, Gander F, Pfister H, Steiger A, Sonntag H, Trenkwalder C, Sonntag A, Hundt W, Wittchen H-U. To what degree does the Composite International Diagnostic Interview (CIDI) correctly identify DSM-IV disorders? Testing validity issues in a clinical sample. Int J Methods Psychiatr Res. 1998;7(3):142-55.

20. Escobar Jl, Rubio-Stipec M, Canino G, Karno M. Somatic symptom index (SSI): a new and abridged somatization construct. Prevalence and epidemiological correlates in two large community samples. J Nerv Ment Dis. 1989;177(3):140-6

21. Kroenke K, Spitzer RL, Williams JB. The PHQ-9: validity of a brief depression severity measure. J Gen Intern Med. 2001;16(9):606-13.

22. Löwe B, Spitzer RL, Zipfel S, Herzog W. Gesundheitsfragebogen für Patienten (PHQ-D). Komplettversion und Kurzform. Testmappe mit Manual, Fragebögen, Schablonen. 2. Auflage. Karlsruhe: Pfizer; 2002.

23. Kroenke K, Spitzer RL, Williams JB, Lowe B. The Patient Health Questionnaire Somatic, Anxiety, and Depressive Symptom Scales: a systematic review. Gen Hosp Psychiatry. 2010;32(4):345-59.

24. Brauns H, Scherer S, Steinmann S. The CASMIN educational classification in international comparative research. A European working book for demographic and socio-economic variables. In: Hoffmeyer-Zlotnik JHP, Wolf C, editors. Advances in Cross-National Comparison. New York: Kluwer; 2003. p. 221-44.

25. Lampert T, Kroll L, Muters S, Stolzenberg H. Measurement of socioeconomic status in the German Health Interview and Examination Survey for Adults (DEGS1). Bundesgesundheitsbl - Gesundheitsforsch - Gesundheitsschutz. 2013;56(5-6):631-6.

26. Dalgard OS, Dowrick C, Lehtinen V, Vazquez-Barquero JL, Casey P, Wilkinson G, Ayuso-Mateos JL, Page H, Dunn G, Group O. Negative life events, social support and gender difference in depression: a multinational community survey with data from the ODIN study. Soc Psychiatry Psychiatr Epidemiol. 2006:41(6):444-51

27. Ellert $U$, Kurth BM. Health related quality of life in adults in Germany: results of the German Health Interview and Examination Survey for Adults (DEGS1). Bundesgesundheitsbl - Gesundheitsforsch - Gesundheitsschutz. 2013;56(5-6): 643-9.

28. Ware JEJ, Kosinski M, Bjorner BJ, Turner-Bowker DM, Gandek B, ME M. User's manual for the SF-36v2 health survey. 2nd ed. Lincoln: Quality Metric Incorported; 2007. 
29. Kamtsiuris $P$, Lange M, Hoffmann R, Schaffrath Rosario A, Dahm S, Kuhnert $R$, Kurth BM. The first wave of the German Health Interview and Examination Survey for Adults (DEGS1): sample design, response, weighting and representativeness. Bundesgesundheitsblatt Gesundheitsforschung Gesundheitsschutz. 2013;56:620-30.

30. Mitchell AJ, Vaze A, Rao S. Clinical diagnosis of depression in primary care: a meta-analysis. Lancet. 2009;374(9690):609-19.

31. Kessler RC, Calabrese JR, Farley PA, Gruber MJ, Jewell MA, Katon W, Keck PE, Nierenberg AA, Sampson NA, Shear MK, et al. Composite International Diagnostic Interview screening scales for DSM-IV anxiety and mood disorders. Psychol Med. 2013;43(8):1625-37.

32. Jacobi F, Hofler M, Siegert J, Mack S, Gerschler A, Scholl L, Busch MA, Hapke U, Maske U, Seiffert I, et al. Twelve-month prevalence, comorbidity and correlates of mental disorders in Germany: the Mental Health Module of the German Health Interview and Examination Survey for Adults (DEGS1-MH). Int J Methods Psychiatr Res. 2014;23(3):304-19.

33. Small GW. Differential diagnoses and assessment of depression in elderly patients. J Clin Psychiatry. 2009;70(12):e47.

34. Mojtabai R. Diagnosing depression in older adults in primary care. N Engl J Med. 2014;370(13):1180-2.

35. O'Connor DW, Parslow RA. Differences in older people's responses to CIDI's depression screening and diagnostic questions may point to age-related bias. J Affect Disord. 2010;125(1-3):361-4.

36. Fiske A, Wetherell JL, Gatz M. Depression in older adults. Annu Rev Clin Psychol. 2009:5:363-89.

37. Alonso J, Angermeyer MC, Bernert S, Bruffaerts R, Brugha TS, Bryson H, de Girolamo G, Graaf R, Demyttenaere K, Gasquet I, et al. Use of mental health services in Europe: results from the European Study of the Epidemiology of Mental Disorders (ESEMeD) project. Acta Psychiatr Scand Suppl. 2004;420:47-54.

38. Wang PS, Aguilar-Gaxiola S, Alonso J, Angermeyer MC, Borges G, Bromet EJ, Bruffaerts R, de Girolamo G, de Graaf R, Gureje O, et al. Use of mental health services for anxiety, mood, and substance disorders in 17 countries in the WHO world mental health surveys. Lancet. 2007;370(9590):841-50.

39. Oliver MI, Pearson N, Coe N, Gunnell D. Help-seeking behaviour in men and women with common mental health problems: cross-sectional study. $\mathrm{Br}$ J Psychiatry. 2005;186:297-301.

40. Burgess PM, Pirkis JE, Slade TN, Johnston AK, Meadows GN, Gunn JM. Service use for mental health problems: findings from the 2007 National Survey of Mental Health and Wellbeing. Aust N Z J Psychiatry. 2009;43(7):615-23.

41. Hickie IB, Luscombe GM, Davenport TA, Burns JM, Highet NJ. Perspectives of young people on depression: awareness, experiences, attitudes and treatment preferences. Early Interv Psychiatry. 2007;1(4):333-9.

42. Rattay P, Butschalowsky H, Rommel A, Prutz F, Jordan S, Nowossadeck E, Domanska O, Kamtsiuris P. [Utilization of outpatient and inpatient health services in Germany: results of the German Health Interview and Examination Survey for Adults (DEGS1)]. Bundesgesundheitsbl Gesundheitsforsch - Gesundheitsschutz. 2013;56(5-6):832-44.

43. Menchetti M, Belvederi Murri M, Bertakis K, Bortolotti B, Berardi D. Recognition and treatment of depression in primary care: effect of patients' presentation and frequency of consultation. J Psychosom Res. 2009:66(4):335-41.

44. Mojtabai R. Unmet need for treatment of major depression in the United States. Psychiatr Serv. 2009:60(3):297-305.

45. Barney LJ, Griffiths KM, Jorm AF, Christensen H. Stigma about depression and its impact on help-seeking intentions. Aust N Z J Psychiatry. 2006:40(1):51-4.

46. Schomerus G, Angermeyer MC. Stigma and its impact on help-seeking for mental disorders: what do we know? Epidemiol Psichiatr Soc. 2008; 17(01):31-7.

47. Schomerus G, Auer C, Rhode D, Luppa M, Freyberger HJ, Schmidt S. Personal stigma, problem appraisal and perceived need for professional help in currently untreated depressed persons. J Affect Disord. 2012; 139(1):94-7.

48. Cepoiu M, McCusker J, Cole MG, Sewitch M, Belzile E, Ciampi A. Recognition of depression by non-psychiatric physicians-a systematic literature review and meta-analysis. J Gen Intern Med. 2008;23(1):25-36.

49. Sielk M, Altiner A, Janssen B, Becker N, de Pilars MP, Abholz HH. Prevalence and diagnosis of depression in primary care. A critical comparison between PHQ-9 and GPs' judgement. Psychiatr Prax. 2009;36(4):169-74.
50. Moller-Leimkuhler AM. Barriers to help-seeking by men: a review of sociocultural and clinical literature with particular reference to depression. J Affect Disord. 2002;71(1-3):1-9.

51. Mack S, Jacobi F, Gerschler A, Strehle J, Hofler M, Busch MA, Maske UE, Hapke U, Seiffert I, Gaebel W, et al. Self-reported utilization of mental health services in the adult German population-evidence for unmet needs? Results of the DEGS1-Mental Health Module (DEGS1-MH). Int J Methods Psychiatr Res. 2014;23(3):289-303.

52. Wilhelm K, Parker G. Sex differences in lifetime depression rates: fact or artefact? Psychol Med. 1994;24(01):97-111.

53. Rosenstrom T, Jokela M. Reconsidering the definition of Major Depression based on Collaborative Psychiatric Epidemiology Surveys. J Affect Disord. 2017;207:38-46.

\section{Submit your next manuscript to BioMed Central and we will help you at every step:}

- We accept pre-submission inquiries

- Our selector tool helps you to find the most relevant journal

- We provide round the clock customer support

- Convenient online submission

- Thorough peer review

- Inclusion in PubMed and all major indexing services

- Maximum visibility for your research

Submit your manuscript at www.biomedcentral.com/submit
) Biomed Central 